\title{
Chaos and Arnold Diffusion in Dynamical Systems
}

\author{
FATHI M. A. SALAM, JERROLD E. MARSDEN, AND PRAVIN P. VARAIYA, FELLOW, IEEE
}

\begin{abstract}
Chaotic motion refers to complicated trajectories in dynamical systems. It occurs even in deterministic systems governed by simple differential equations and its presence has been experimentally verified for many systems in several disciplines. A technique due to Melnikov provides an analytical tool for measuring chaos caused by horseshoes in certain systems.. The phenomenon .of . Arnold diffusion is another type of complicated behavior. Since 1964, it has been playing an important role for Hamiltonian systems in physics. We present a tutorial treatment of this work and its place in dynamical systems theory, with an emphasis on results that can be checked in specific systems.

A generalization of the Melnikov technique has been recently developed to treat $n$-degree of freedom Hamiltonian systems when $n>3$. We extend the Melnikov technique to certain non-I Iamiltonian systems of ordinary differential equations. The extension is made with a view to applications in the physical sciences and engineering.
\end{abstract}

\section{INTRODUCTION}

$\mathrm{C}$ HAOS refers to the unpredictable, seemingly random, motion of trajectories of a dynamical system. The dynamical system may be described by a differential equation or a map, i.e., a discrete-time system. It was first observed by Cartwright, Littlewood, and Levinson [11] in the two-dimensional Van Der Pol equation with a forcing term. Smale [42] succeeded in devising a map, called the horseshoe map, which geometrically exhibits the complicated dynamics of a random motion. It was later established that a horseshoe is indeed embedded in certain dynamical systems displaying chaotic behavior. There is of course more to the subject of chaos than horseshoes, although horseshoes can be involved in the formation of "strange attractors" such as in the Lorenz attractor, see Guckenheimer [17], Mees and Sparrow [33], and Sparrow [43]. Melnikov [24] developed a technique which measures the presence of chaos in certain periodically forced two dimensional systems of ordinary differential equations.

In 1964, Arnold [5] announced another type of complicated motion subsequently known as Arnold Diffusion. The physical interpretation of Arnold diffusion is that, given enough time, chaotic energy transfer occurs between subsystems of a conserved system. Furthermore, one may incorporate certain positive and negative damping to allow for the nonconservative case. This phenomenon of chaotic energy transfer is of great importance in applications.

Manuscript received January 10, 1982; revised February 10, 1983. This work is supported in part by DOE under Contract DE-ASOI-78ET29135 and under Contract DE-AT03-82 ER 12097.

F. M. A. Salam was with the University of California, Berkeley, CA. He is now with the Department of Mechanical Engineering and Mechanics, Drexcl University, Philadelphia, PA 19104

J. E. Marsden is with the Department of Mathematics, University of California, Berkeley, CA 94720.

P. P. Varaiya is with the Department of Electrical Engineering, University of California, Berkeley, CA 94720.
Chaos and Arnold diffusion are a result of certain global qualitative features of a system's phase portrait. These features can arise under small perturbations of a simpler system and hence can be thought of as belonging to the general field of bifurcation theory.

Our contribution is theoretical in nature and is described as follows: we indicate a suggestive analogy between the phenomenon of Arnold diffusion and that of the $\Omega$-decomposition theorem of Smale [42], [11]. This permits us to associate Arnold diffusion with a general class of diffusion, namely that of the $\Omega$-decomposition theorem. We extend the recent result of Holmes and Marsden [27] on the Melnikov technique and Arnold diffusion to certain nonHamiltonian systems. Their result extends the work of Arnold [5] to general $n$-degree of freedom Hamiltonian where $n>2$.

The Melnikov technique introduces an integral function that measures the first variation of the separation between the perturbed stable and unstable manifolds of a hyperbolic point. For improved accuracy of the separation's measure, one can determine, in principle, as many terms of the Taylor expansion of the separation as required.

This paper is organized in the following way; in Section II, an overview of dynamical systems is presented from the point of view of structural stability. The horseshoe and other mappings are introduced and we indicate the new phenomena in dynamical systems that they reveal. We end the section with the $\Omega$-decomposition theorem of Smale which helps one understand the general phenomenon of diffusion.

Section III treats the existing results on chaos and the Melnikov measuring technique. We point out how the accuracy of the method can be improved by considering more terms of the expansion of the separation.

Section IV first reviews the work of Holmes and Marsden [26], [27] which extends the work of Arnold [5] to more general $n$-degree of freedom Hamiltonian systems where $n>2$. This is based on the development of a vector Melnikov function as an extension of the scalar one. Then as extension of the Melnikov vector version to include certain non-Hamiltonian systems is introduced.

One of our aims in writing this paper is to acquaint the readers with some aspects of dynamical systems that will be important in the treatment of power systems [45], [2], to be published.

\section{AN OVerview of DyNAmical Systems}

The mathematical literature on dynamical systems is immensely rich and diversified. It has developed a large 
number of definitions and concepts. Here we shall present a selective review, introducing concepts as needed, so that we can better pursue our main theme. In what follows, we shall consider both diffeomorphisms (discrete-time systems) and vector fields of differential equations. It is known that results on diffeomorphisms can be extended to vector fields (or more precisely flows) by suspension techniques (see Smale [42]). Results on diffeomorphisms are often applied to flows by considering Poincaré maps, as is explained in Section III-3.3. In this section we shall mostly discuss diffeomorphisms.

In the early 1960's there was extensive work on dynamical systems. The aim was to classify the generic dynamical behavior of systems. A property is said to be generic if this property is possessed by an (open) dense set of maps. A less restrictive concept is that of properties of dynamical systems which persist under small perturbations. A flow whose whole phase portraits is topologically unchanged by perturbations is called structurally stable.

Our main aim here is to review the development of the notion of structural stability of diffeomorphisms (respectively flows) which led, indirectly at least, to the discovery of dynamical behavior of a complicated nature. For a more complete exposition, see Smale [42], Moser [35], Newhouse [36], Chillingworth [11], Nitecki [37], and Abraham and Marsden [3].

\subsection{Structural Stability}

Peixoto (see [42], [35]) took a first crucial step in what would become later an important development. His result, first given for vector fields, gives criteria for structural stability on two-dimensional manifolds. To elucidate these results, we introduce the wandering set.

Let $\boldsymbol{M}$ be a compact manifold, $f \in \operatorname{diff}(\boldsymbol{M})$ the space of diffeomorphisms, and $\Phi_{t}$ be a flow on $\boldsymbol{M}$. A point $p \in \boldsymbol{M}$ is a wandering point for $f$ if there is a neighborhood $U$ of $p$ such that $\bigcup_{|m|>0} f^{m}(U) \cap U=\varnothing$. A point $q \in \boldsymbol{M}$ is a wandering point for the flow $\Phi_{l}$ if there exist a neighborhood $V$ of $q$ in $M$ such that $\left(U_{|z|>t_{0}} \Phi_{t}(V)\right) \cap V=\varnothing$ for some $t_{0}>0$. The set of points which are not wandering is the nonwandering set. We now state Peixoto's result

Theorem 2.1 [Peixoto] (structural stability on 2-manifolds):

A $C^{k}, k \geqslant 1$, vector field on a two-dimensional manifold $\boldsymbol{M}$ with $\Omega$ as its nonwandering set is structurally stable if and only if it satisfies the following conditions:

(1) The number of fixed points and periodic orbits is finite, and each is hyperbolic, i.e., the eigenvalues of the Jacobian of the vector field are not equal to one in absolute value.

(2) There are no trajectories joining saddle points.

(3) $\Omega$ consists of fixed and periodic orbits only.

Moreover, if $\boldsymbol{M}$ is orientable then the set of such vector fields is open and dense in $\boldsymbol{X}^{k}(\boldsymbol{M})$.

Smale and others attempted to extend the theorem above to dimensions higher than two. Smale considered a diffeomorphism $f \in \operatorname{diff}^{k}(\boldsymbol{M})$, where $\boldsymbol{M}$ is a compact manifold, which satisfies the conditions of Theorem 2.1 with condi- tion (2) replaced by a transversality condition. More specifically: (1) $\Omega$, the nonwandering set, is finite. (2) The periodic points of $f$ are hyperbolic. (3) (Transversal intersection condition) For any points $p, q \in \Omega$, the stable manifold $W^{s}(p)$ and the unstable manifold $W^{u}(q)$ intersect transversally.

Systems that satisfy the three conditions cited above have been named Morse-Smale systems (or, for short $M-S$ systems). Furthermore, they obey a set of inequalities (called the Morse-Smale inequalities). These inequalities translate to a set of topological constraints on the possible number of fixed points or periodic orbits for any $M-S$ system. It was then thought that $M-S$ systems would provide a logical extension to Peixoto's theorem, so the following conjectures were proclaimed.

Conjecture (1): A system is structurally stable if and only if it is an $M-S$ system.

Conjecture (2): $M-S$ systems are dense in $\operatorname{diff}^{k}(\boldsymbol{M})$. Or another possibility,

Conjecture (3): Structurally stable systems are dense in $\operatorname{diff}^{k}(\boldsymbol{M})$.

These conjectures were quickly proved incorrect. Conjecture (1) is partly true; Palis proved that $M-S$ systems form an open set in $\operatorname{diff}^{1}(\boldsymbol{M})$. Then Palis and Smale proved that $M-S$ systems are structurally stable. The reverse (i.e., structural stability implies $M-S$ ) has been found to be false in general. Conjectures (2) and (3) are also false. For further information, see Abraham and Marsden [3, sec. 7.5].

The failure of conjectures (1) and (2) suggested the existence of a more complicated behavior than was already known. Furthermore, it implied that these complicated phenomena could be structurally stable. We now present the famous two examples that negated the cited first two conjectures. One should note that there are other examples which serve the same purpose, e.g, the tent mapping, and the Henon mapping (see Lanford [30], Lichtenberg and Lieberman [32], Henon [21]).

\subsubsection{Example 1. Hyperbolic Toral Automorphism} (Anosov Diffeomorphisms)

Let $A$ be a $2 \times 2$ matrix with integer elements and determinant $= \pm 1$. Specifically let

$$
A=\left[\begin{array}{ll}
1 & 2 \\
1 & 1
\end{array}\right]
$$

with zero as a saddle fixed point and eigenvalues $\lambda=(1$ $+\sqrt{2})$ and $\mu=(1-\sqrt{2})$.

The saddle's stable and unstable manifolds are the $1-d$ eigenspaces corresponding, respectively, to $\mu$ and $\lambda$. $A$ can be considered as a linear transformation that preserves the lattice $L$, in $R^{2}$, of points with integer coordinates.

The map $A$ defines an induced diffeomorphism on the torus $T^{2}$ (which is the quotient space $R^{2} / L$ ). Denote this induced map by $f$, so $f: T^{2} \rightarrow T^{2}$.

Since the saddle point 0 in $R^{2}$ is hyperbolic, so is its image $p$ on the torus. The eigenspace in $R^{2}$ corresponding to $\lambda(\mu)$ is mapped to the unstable (stable) manifold 
$W^{u}(p)\left(W^{s}(p)\right)$, which winds densely around the torus. I'his map from $R^{2}$ to $T^{2}$ is a one-to-one immersion.

One should note that the homoclinic points $\{x \mid x \in$ $W^{s}(p) \cap W^{u}(p)$ and $\left.x \neq p\right\}$ are dense in $T^{2}$. This example has the following properties (see Smale [42, p. 758]).

(1) The periodic points are dense in $T^{2}$. The nonwandering set $\Omega(f)$ which is closed is, therefore, the whole of $T^{2}$.

(2) The homoclinic points have orbits that are dense in $T^{2}$.

(3) $f$ is structurally stable.

Thus the toral automorphism is a structurally stable map but not an $M-S$ map since, among other things, it possesses an infinite number of periodic points.

\subsubsection{Example 2. Smale's Horseshoe map}

Let $Q$ be the square $[-1,1]^{2}$ in $R^{2}$. Consider the open neighborhood $(-1-\epsilon, 1+\epsilon)^{2}, \epsilon>0$, and attach (open) half circles to each of its left- and right-hand sides as in (Fig. 1(a)) and denote the resulting (open) domain $Q^{+}$. Define a map $T$ by the following geometrical operations. (a) Stretch $Q^{+}$horizontally. (b) Bend it in the middle to take the form of a horseshoe as in (Fig. 1(b)). (Note that points $A, B, C, D$, map to points $\bar{A}, \bar{B}, \bar{C}, \bar{D}$, respectively.) (c) Superimpose the resulting horseshoe upon the domain $Q^{+}$as in (Fig. 1(c)).

It can be shown by using pasting techniques (see Boothby [9]) that the previous operations can be performed by means of a smooth map $T$. Furthermore $T: Q^{+} \rightarrow Q^{+}$is 1-1 but not onto. To obtain a global map one may compactify the domain by a one point compactification (the point $\infty$ ) and, using the stereographic map, one can show that the domain is a diffeomorphism of the two sphere $S^{2}$ with the point $\{\infty\}$ acting as a source. Note that there exists an attracting fixed point $q^{0}$ (Fig. 1(c)). Now observe that the image of the vertical strip $\Delta_{0}\left(\Delta_{1}\right)$, under $T$, is the horizontal strip $\bar{\Delta}_{0}=T\left(\Delta_{0}\right)\left(\bar{\Delta}_{1}=T\left(\Delta_{1}\right)\right)$ as shown in (Fig. 1(d)). Thus on the square $Q$ one may consider the effect of $T$ to be the same as the following "linearized" maps: $\alpha>1>\beta>0$

$$
\bar{T}_{x}^{0}=\left[\begin{array}{ll}
\alpha & 0 \\
0 & \beta
\end{array}\right] x+\gamma^{0}
$$

where $\bar{T}_{x}^{0}$ is the map $T$ restricted to the domain $\Delta_{0}$ with $x \in \Delta_{0}$, and $\gamma^{0}$ is a constant vector, and

$$
\bar{T}_{y}^{1}=\left[\begin{array}{cc}
-\alpha & 0 \\
0 & -\beta
\end{array}\right] y+\gamma^{1}
$$

where $\bar{T}_{y}^{1}$ is the map $T$ restricted to the strip $\Delta_{1}$ with $y \in \Delta_{1}$ and $\gamma^{\mathbf{l}}$ is a constant vector.

A candidate for the basin of attraction of the fixed point $q^{0}$ is the whole of $Q^{+}$minus the two vertical strips $\Delta_{0}$ and $\Delta_{1}$ (the shaded region in Fig. 1(e)). Consider the iterate $T^{2}=T \cdot T$ on $Q^{+}$with its image superimposed on $Q^{+}$is as shown in (Fig. 1(f)). Then consider the following intersection:

$$
Q \cap T\left(Q^{+}\right) \cap T\left(T\left(Q^{+}\right)\right) \cap T\left(T\left(T\left(Q^{+}\right)\right)\right) \cap \cdots
$$

which equals

$$
Q \cap T\left(Q^{+}\right) \cap T^{2}\left(Q^{+}\right) \cap T^{3}\left(Q^{+}\right) \cap \cdots
$$

One can show that this intersection is the set $I \times C$ (Fig. $1(\mathrm{~g}))$ where $C$ is a homeomorphism of the Cantor set and $I$ is the interval $[-1,1]$. Similarly for the inverse mapping $T^{-1}$ one can show that

$$
Q \cap T^{-1}(Q+) \cap T^{-2}(Q+) \cap \cdots
$$

is the set $C \times I$ where $C$ and $I$ are as defined above. Therefore, the nonwandering set of $T$, denoted $\Omega$, is the intersection of the sets (a) and (b) above, i.e., $I \times \underline{C} \cap \underline{C}$ $\times I=\underline{C} \times \underline{C}$ which is homeomorphic to the Cantor set [36], [37], [42]. Hence the nonwandering set is a Cantor set which is closed and contains infinite number of points. Moreover, the nonwandering set is hyperbolic (recall the maps $\bar{T}_{x}^{0}$ and $\bar{T}_{y}^{1}$ ) and the periodic points are dense in $\Omega$ (see [36], [11]). It follows that the map $T$ is not an $M-S$ map.

The essential conclusion here is that $T$ is structurally stable and hence disproves conjecture (1) immediately (see Smale [42]) and consequently conjecture (2) fails as well.

The two examples we have discussed have dynamics exhibiting statistical behavior (see Moser [35]). It will be useful to discuss the shift automorphism to comprehend the orbit behavior of these complicated systems.

\subsubsection{The Shift Automorphism}

Let $A=\left\{a_{1}, a_{2}, \cdots, a_{k}\right\}$ be a finite alphabet. Consider the set $S$ of all doubly infinite sequences $s=\left(\cdots, s_{-2}\right.$, $\left.s_{-1}, s_{0} ; s_{1}, s_{2}, \cdots\right)$ such that $s_{i} \in A$. We construct a topology on $S$ as follows. Define a basis at any $s^{*}=$ $\left(\cdots, s_{-1}^{*}, s_{0}^{*} ; s_{1}^{*}, s_{2}^{*}, \cdots\right)$ by

$$
U_{j}=\left\{s \in S \mid s_{k}=s_{k}^{*} ; \text { for all }|k|<j\right\} \quad \text { for } j=1,2, \cdots \text {. }
$$

With this topology, $S$ becomes a topological space. One now defines the shift homeomorphism $\sigma: S \rightarrow S$ by $(\sigma(s))_{k}$ $=s_{k-1}$, i.e., one simply shifts the sequence to the right.

\subsubsection{The Shift and the Horseshoe}

In the special case in which $A$ contains only two members $a_{1}$ and $a_{2}$, denoted simply zero and one, we can identify the vertical strip $\Delta_{0}\left(\Delta_{1}\right)$, in the horseshoe mapping, with the symbol 0 (1). Thus starting with a nonwandering point $x$ its (past and future) orbit is described by the doubly infinite sequence $\left(\cdots, T^{-1}(x), x, T(x)\right.$, $\left.T^{2}(x), \cdots\right)$.

If $x \in \Omega$ resides in the vertical strip $\Delta_{0}\left(\Delta_{1}\right)$, then $T(x)$ may reside in either $\Delta_{0}$ or $\Delta_{1}$ depending on the (unique) orbit of the (initial) point $x$. Hence we can replace the sequence above with a shift sequence in the alphabet $\{0,1\}$. In fact it has been shown (see [42], [35], [36]) that the correspondence of the orbits of the map $T$, restricted to the nonwandering set $\Omega$, and the shift automorphism elements constitute a homeomorphism.

With the help of the shift it is easy to verify that the periodic points are dense in the nonwandering set $\Omega$ of $T$ : in fact the periodic sequences correspond to periodic points.

Finally we consider the notion of basic sets and what is called the $\Omega$ decomposition theorem.

\subsection{The $O M E G A-$ Decomposition Theorem}

We first introduce a general definition of hyperbolicity, generalizing the notion of a hyperbolic fixed point. 


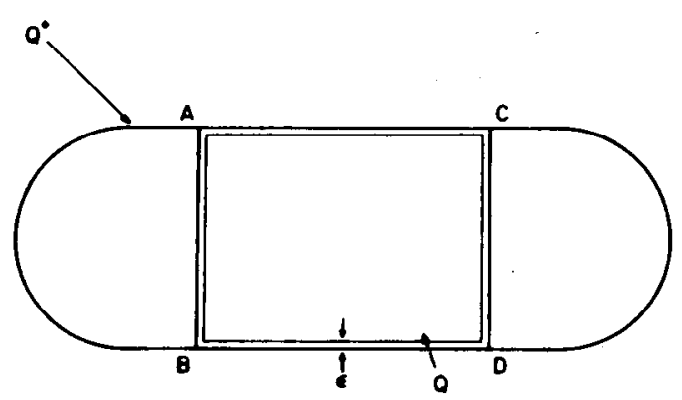

(a)

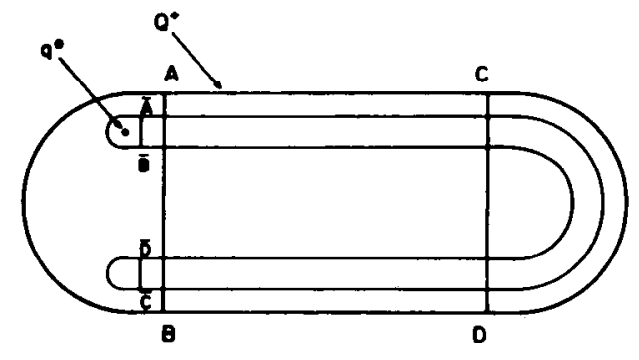

(c)

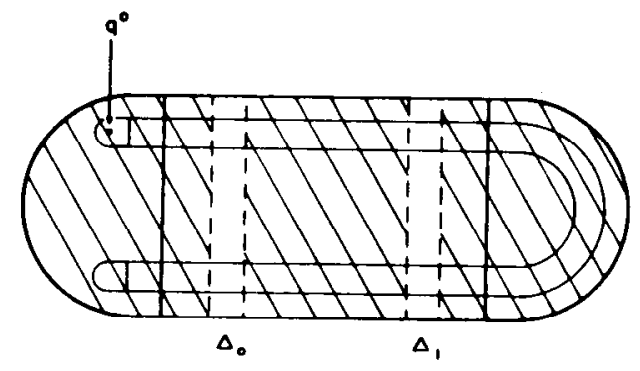

(e)

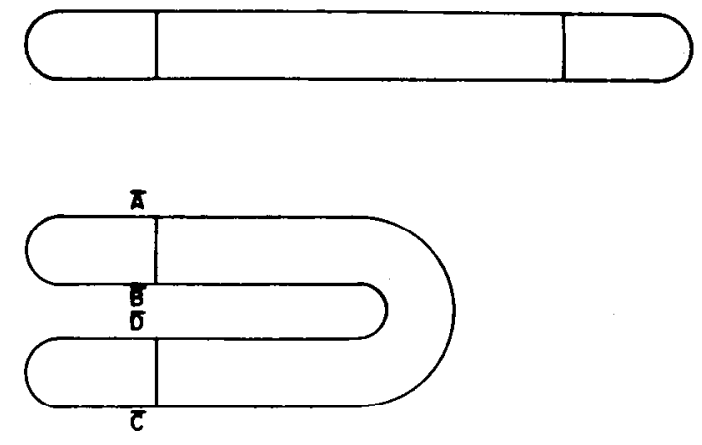

(b).

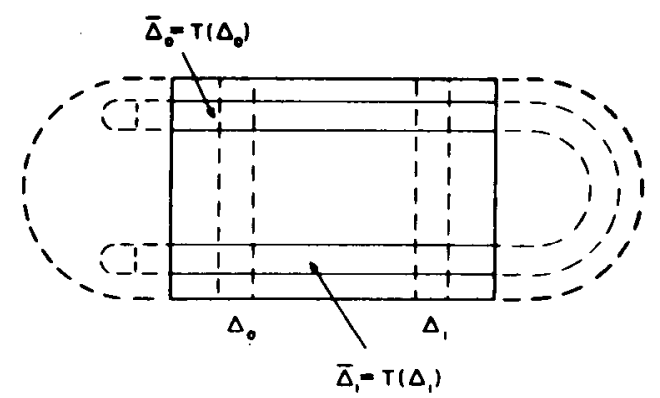

(d)

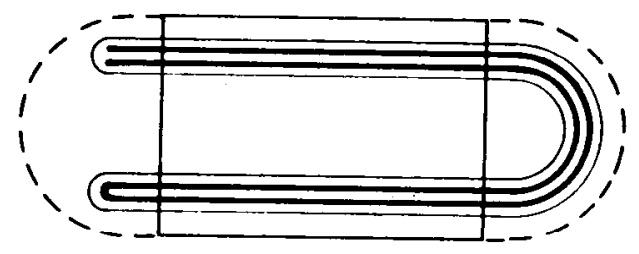

(f)

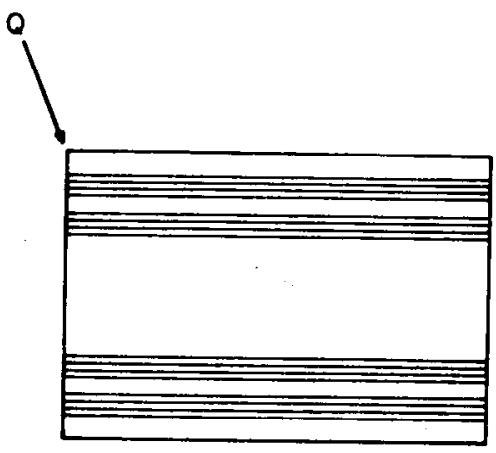

(g) Fig. 1. (a)-(d) The domain of the horseshoe mapping. (e)-(g) The
nonwandering set of the horseshoe.

Definition [42], [36], [37]: Let $\Lambda$ be any closed invariant set for the diffeomorphism $f$ on a compact manifold $\boldsymbol{M} . \Lambda$ is hyperbolic if for each $p \in \Lambda$ there is a splitting of the tangent space $T_{p} \Lambda$, into a direct sum of two subspaces, which varies continuously with $p$. Moreover, there exists a (Riemannian) norm $|\cdot|$ and constants $c, C$, and $\lambda<1$ such that the following holds:

(1) $T_{p} f\left(E_{p}^{s}\right)=E_{f(p)}^{s} \quad$ and $\quad T_{p} f\left(E_{p}^{u}\right)=E_{f(p)}^{u}$
(2)

$$
\begin{array}{ll}
\left|T_{p} f^{n}(v)\right|_{f^{n}(p)}<C \lambda^{n}|v|_{p} & \text { for all } v \in E_{p}^{s} \text { and } \\
\left|T_{p} f^{n}(v)\right|_{f^{n}(p)}>c \lambda^{-n}|v|_{p} & \text { for all } v \in E_{p}^{u}
\end{array}
$$
for every positive integer $n$.

We quote the Generalized Stable Manifold Theorem due to Hirsch, Pugh, and Shub [22].

Theorem 2.2: Let $x$ be an element of the invariant hyperbolic set $\Omega$ of $f \in \operatorname{diff}^{k}(M)$. Then 


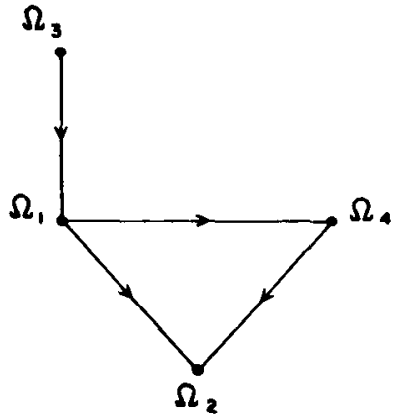

Fig. 2. A labeled diagram of the Omega decomposition Theorem.

(i) For each $x$, the stable manifold $W^{s}(x)$ is an immersed copy of $R^{s}$, with $E_{x}^{s}$ as its tangent space at $x$.

(ii) As $x$ runs through $\Omega$ all the $W^{s}(x)$ fit together to give a continuously varying family (at least locally near $\Omega$ ).

(iii) This family is invariant, i.e., $\boldsymbol{W}^{s}(f(x))=f\left(\boldsymbol{W}^{s}(x)\right)$. Similar results hold for the unstable manifold.

Wè now state the Structural Stability Theorem [11], which generalizes Peixoto's two-dimensional theorem. First we introduce

Axiom $A$ (Smale [42]): A diffeomorphism $f$ is said to satisfy Axiom $\mathrm{A}$ if the following holds.

(a) The nonwandering set $\Omega$ of $f$ is hyperbolic.

(b) The periodic points of $f$ are dense in $\Omega$.

We remark that only on the plane (dimension 2), condition (a) implics condition (b).

Theorem 2.3 (Robbin [11], [37]) (Structural stability theorem): If $\boldsymbol{M}$ is compact and the diffeomorphism $f \in$ $\operatorname{diff}^{k}(\boldsymbol{M})$ satisfies Axiom A and the transversality condition, i.e., the (generalized) stable and unstable manifolds intersect transversally, then $f$ is structurally stable.

The following theorem captures many of the abstract concepts we are concerned with.

Theorem 2.4 [42] ( $\Omega$-Decomposition of Diffeomorphisms): Suppose $f: \boldsymbol{M} \rightarrow \boldsymbol{M}$ satisfies Axiom A. Then there is a unique way of writing $\Omega$ as the finite union of disjoint, closed, invariant, indecomposable subsets: $\Omega=\Omega_{1} \cup \cdots \cup$ $\Omega_{m}$. Furthermore, on each subset $\Omega_{k} f$ is topologically transitive, i.e., it has an orbit dense in $\Omega_{k}$.

The sets $\Omega_{k}$ are called hasic sets. We note that the manifold $\boldsymbol{M}$ decomposes into invariant sets for $f$, i.e., $\boldsymbol{M}=\cup_{k-1}^{m} \boldsymbol{W}^{s}\left(\Omega_{k}\right)$.

As in Smale [42], Nitecki [37], Newhouse [36], and Chillingworth [11], if Axiom A and the no-cycle condition (see Smale [42]) are both satisfied, then we may denote $\Omega_{i} \leqslant \Omega_{j}$ if $\boldsymbol{W}^{s}\left(\Omega_{i}\right) \cap W^{u}\left(\Omega_{j}\right) \neq \varnothing$. Say that $\Omega_{i_{1}} \leqslant \Omega_{i_{2}} \leqslant \cdots \leqslant$ $\Omega_{i_{n}}$ is a maximal chain if the $\Omega_{i_{k}}$ 's are distinct and $n$ is maximal. In fact one may draw the labeled diagram (Fig. 2) where each vertex corresponds to an $\Omega_{k}$ and each directed 1-simplex (or branch) joins consecutive vertices of maximal chains. This diagram is invariant under small perturbations of $f$. We note that in this graph there are no cycles, a consequence of transversal intersections of stable and unstable manifolds (see Smale [42] and Newhouse [36]). $\Omega_{2}$ in the labeled graph of (Fig. 2) assumes the role of an attractor.
With this selective review of dynamical systems we come to a point of special interest. In the maximal chains a solution orbit may start from the unstable manifold of an invariant basic set $W^{u}\left(\Omega_{j}\right)$, and as time progresses, it diffuses to the stable manifold of another basic set $W^{s}\left(\Omega_{i}\right)$. Thus maximal chains express a general diffusion.

\section{Chaos and Its Measuring Techniques}

This last section introduced two examples of structurally stable complicated behavior of dynamical systems of diffeomorphisms. Here we are interested in how one can determine whether a given dynamical system possesses such complicated behavior. Our attention is focused on methods relevant to homoclinic type chaos. We begin with a discussion of the physical meaning (and history) of chaos.

\subsection{Introduction and Physical Meaning}

Chaos in a dynamical system refers to the complex (statistical-like) behavior of a solution orbit of the system described (either a differential equation or a map). It was first observed in the two-dimensional system of the Van Der Pol equation, with a forcing term, by Cartwright, Littlewood, and Levinson. Its discovery led Smale to express its behavior by the horseshoe mapping of the previous section. For a typical system with chaotic orbits, there will be a neighborhood $U$ in phase space such that, over time, the separation between (close) points in this neighborhood $U$ will grow at an exponential rate. That is to say, there are points in $U$ which will have totally different fate no matter how small $U$ is. Levinson's example exhibits this last property as does Smale's horseshoe.

Transversal intersections of the stable and unstable manifolds of a saddle point of the Van Der Pol equation accounts for this behavior. More precisely, it is the presence of homoclinic points of the transversal intersection of the manifolds of the induced Poincare map that are "responsible" for the chaos. The existence of the homoclinic points implies that the structure of the horseshoe is embedded near these points. One can actually reveal the connection between the horseshoe map and the transversal intersection of (stable and unstable) manifolds as we shall explain in Section III.3.1 (see Smale [42], Newhouse [36], Holmes and Marsden [26], and Greenspan and Holmes [15]). Then, knowing that the horseshoe is structurally stable, leads to the conclusion that chaos can survive sufficiently small perturbations. We shall illustrate these concepts for the two-dimensional (time-varying) case next.

\subsection{Measuring Chaos}

There exist different variants of one method of testing for the presence of chaos in a given (dynamical system) of differential equations. The basic method is due to Melnikov [24]. Holmes [24], Chow, Hale, and Mallet-Paret [13], Greenspan and Holmes [15], Holmes and Marsden [25], [26] contributed to its elaboration and development. Chow et al. [13] gave conditions leading to a complete picture of a bifurcation diagram for a specific (time-vary- 
ing) two-dimensional example. Holmes and Marsden [26] developed a version of the Melnikov method for twodegree of freedom Hamiltonian systems composed of a cross-product of a subsystem possessing a homoclinic orbit and a nonlinear oscillator. They further extended their results to higher than 2-degree of freedom Hamiltonian systems where Arnold diffusion may emerge [27]. There Hamiltonian system is that of a cross-product of one possessing a homoclinic orbit and several nonlinear oscillators. We refer to Lichtenberg and Lieberman [32] for experimental and physical aspects.

\subsubsection{Poincare Maps and Connection to the Horseshoe}

Consider the nonautonomous differential equation

$$
\dot{x}=f(x)+\mu g(x, t)
$$

where $x \in R^{2}$, with $g(x, t)=g(x, t+T)$ and the perturbation parameter $\mu>0$, is small. Assume that (3.3.1) possesses a flow (the precise conditions will be stated in the next subsection). We suspend (3.3.1) over the space $R^{2} \times S^{1}$ where $S^{1}=R / T$ is the circle of length $T$.

$$
\begin{aligned}
& \dot{x}=f(x)+\mu g(x, \vartheta), \quad(x, \vartheta) \in R^{2} \times S^{1} \\
& \dot{\vartheta}=1 .
\end{aligned}
$$

Let $\mu=0$ and obtain the unperturbed differential equation

$$
\begin{aligned}
& \dot{x}=f(x) \\
& \dot{y}=1 .
\end{aligned}
$$

We study the induced map of this differential equation - the Poincaré map. This procedure reduces the domain of the system by one dimension, and more importantly it captures the same dynamics as the differential equation. Consider the cross.section for the flow in the space $R^{2} \times S^{1}$ as in (Fig. 3(a)):

$$
\Sigma^{t_{0}}=\left\{(x, \vartheta) \in R^{2} \times S^{1} \mid \vartheta=t_{0} \in[0, T)\right\}
$$

The Poincaré map for (3.3.2) $P_{\mu}^{t_{0}}: \Sigma^{t_{0}} \rightarrow \Sigma^{t_{0}}$ is defined by

$$
P_{\mu}^{t_{0}}\left(x_{\mu}\left(t_{0}\right)\right)=\Pi\left(x_{\mu}\left(T+t_{0}, t_{0}\right), \vartheta(T)\right)=x_{\mu}\left(T+t_{0}, t_{0}\right)
$$

where $x_{\mu}\left(t, t_{0}\right)$ is the solution of (3.3.1). $\Pi$ is the projection map in the first factor.

For $\mu=0$ one similarly obtains the Poincare map for the unperturbed system (3.3.3) (see Fig. 3(b)) as $P_{0}^{t_{0}}\left(x_{0}(0)\right)=$ $x_{0}(T)$.

Observe that the unperturbed Poincare map $P_{0}^{t_{0}}$ is the same on every section $\Sigma^{t_{0}}$ for $t_{0} \in[0, T)$, whereas the perturbed Poincare map may differ on different sections. All the perturbed Poincare maps are diffeomorphic and furthermore $P_{\mu}^{t_{0}+n T}=P_{\mu}^{t_{0}}$ (for simplicity we drop the superscript $t_{0}$ of the Poincare maps when it is easily understood).

Recall from Scction II that, for a (Poincaré) map $P$, a homoclinic point $q$ to a hyperbolic saddle point $p$ of $P$ is a point whose orbit approaches $p$ under both forward and backward iterates of $P$. This is so since $q$ lies on both stable and unstable manifolds of $p$, i.e., $\lim _{n \rightarrow \infty} P^{n}(q)=$ $\lim _{n \rightarrow \infty} P^{-n}(q)=p$. Hence $q \in W^{u}(p) \cap W^{s}(p)$. If the intersection is transversal in $R^{2}$, one writes $q \in$ $W^{u}(p) 历 W^{s}(p)$. Note that the existence of one such point $q$ in the intersection implies that $P^{n}(q), n \in Z$, are also in the intersection. We now state the following Lemma which is due to Palis.

$\lambda$-Lemma [15]: Let $P$ be a $C^{1}$ diffeomorphism with a hyperbolic fixed point $p$ and let $D^{u}$ be a $u$-disc in the unstable manifold $W^{u}(p)$. Let $\Delta$ be a $u$-disc meeting the stable manifold $W^{s}(p)$ transversally at some point $x$. Then $\cup_{n \geqslant 0} P^{n}(\Delta)$ contains $u$-discs arbitrarily $C^{1}$ close to $D^{u}$.

A $u$-disc is simply a disc of the unstable manifold in the subspace topology. The $\lambda$-lemma asserts that $W^{u}(p)$ accumulates upon itself and thus leading to complicated dynamics (see (Fig. 3(c)). The existence of a transverse homoclinic point $q$ of the Poincare map (and hence infinitely many of such points) further implies that horseshoe dynamics are present nearby these homoclinic points. Let us state the homoclinic theorem, due to Smale (see Moser [35] and Newhouse [36] for details).

Theorem 3.3: Let $P$ be a $C^{1}$ diffeomorphism with a hyperbolic periodic point $p$ having a transverse homoclinic point $q$. There is an integer $m>0$ such that $f^{m}$ has a closed invariant set $\Omega$ containing $q$ and $p$ so that $f^{m} \mid \Omega$ is topologically equivalent to the shift automorphism. Moreover, $\Omega$ is a hyperbolic set for $f^{m}$.

Newhouse [36] extended Palis' result to admit full neighborhoods of the domain. One may, therefore, consider a neighborhood $N_{\delta}^{s}$ adjacent to the stable manifold and contained in a compact square $Q$. Let $P^{n}\left(N_{\delta}^{s}\right)$ be the image of this neighborhood for some integer $n$ as in Fig. 3(d). It is then observed that $P^{n}$, restricted to the (compact) square, defines a homeomorphism to the horseshoe map discussed in Section II; for more details see [42], [36], [35], [26], [37].

\subsubsection{The Melnikov Technique}

Continuing the development of the previous subsection, we first restate equation (3.3.2), the perturbed system,

$$
\begin{aligned}
& \dot{x}=f(x)+\mu g(x, \vartheta) \\
& \dot{\vartheta}=1
\end{aligned}
$$

where $(x, \vartheta) \in R^{2} \times S^{1}$ and $\mu>0$. Setting $\mu=0$ one obtains the unperturbed differential equations

$$
\begin{aligned}
& \dot{x}=f(x) \\
& \dot{\vartheta}=1 .
\end{aligned}
$$

We now state our assumptions precisely;

(A1) The autonomous equation $\dot{x}=f(x)$ possesses a hyperbolic saddle point $p_{0}$ which is connected to itself by its associated homoclinic orbit

$$
\bar{\Gamma}_{0}=\left\{\bar{x}_{0}(t)\right\}=W^{u}\left(p_{0}\right) \cap W^{s}\left(p_{0}\right) .
$$

(A2) The vectors $f$ and $g$ are $C^{k}, k \geqslant 1$ and bounded on bounded sets. $g$ is $T$-periodic in $\vartheta$.

(A3) Solutions of (3.3.5) exist and are defined over an open neighborhood $D$ such that its closure $\bar{D}$ contains the saddle $p_{0}$ and the homoclinic orbit $\bar{\Gamma}_{0}$.

These assumptions guarantee well-defined solutions of both the perturbed and unperturbed system over the compact domain $\bar{D}$. 


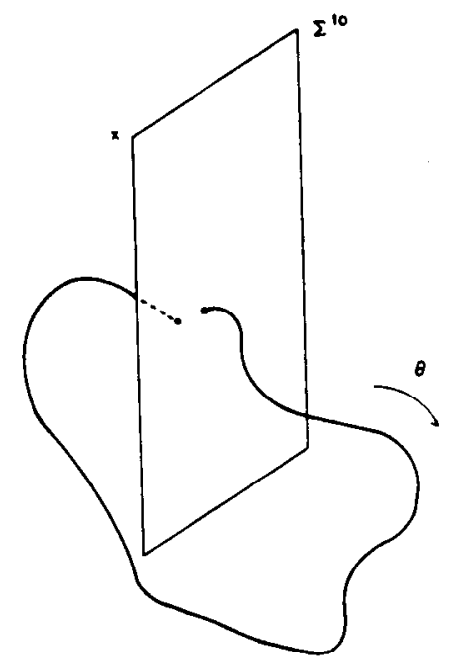

(a)

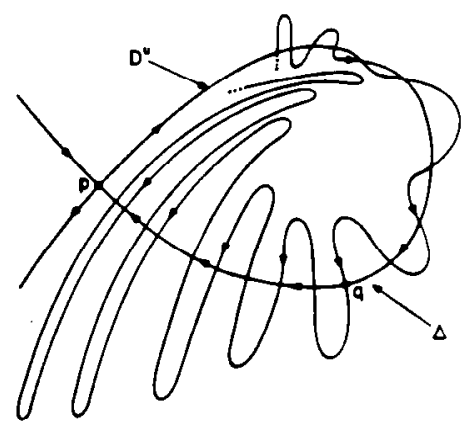

(c)

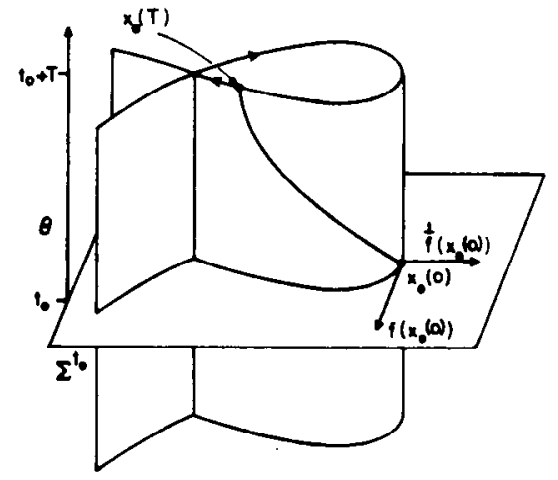

(e)

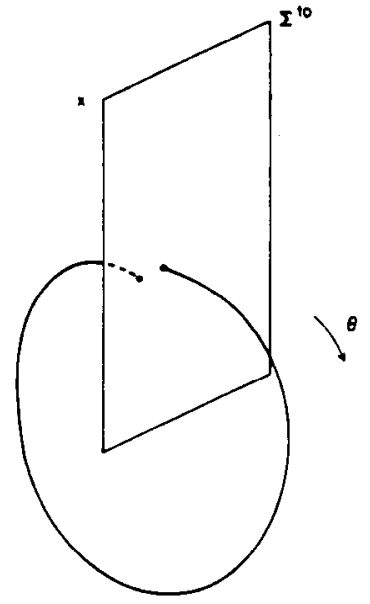

(b)

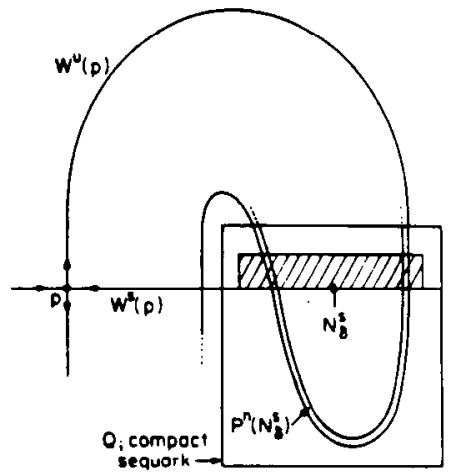

(d)

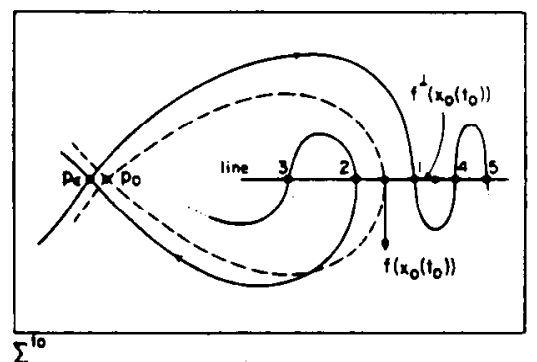

(f)

Fig. 3(a). The space of trajectories of the perturbed system. (b) The space of trajectories of the unperturbed system. (c) The accumulation of a manifold upon itself. (d) An embedded horseshoe. (e) Trajectories on the homoclinic manifold. (f) Measuring the separation between-stable and unstable manifolds.

We consider the trajectories of the unperturbed system (3.3.5) in $R^{2} \times R$ (i.e., in the state variables $(x, \vartheta)$ ). The homoclinic invariant manifold $\left(\bar{\Gamma}_{0} \times R\right)$ takes a cylindrical shape (see Fig. 3(e)). Let $\Sigma^{t_{0}}$ be a section at time $t=t_{0}$, a trajectory on the manifold $\left(\bar{\Gamma}_{0} \times R\right)$ beginning at a point $x(0)$ on the section $\Sigma^{t_{0}}$, spirals once around $\bar{\Gamma}_{0} \times R$ and eventually converges to the saddle point, $p_{0}$, as $t \rightarrow \pm \infty$. The following two lemmas give some properties of the perturbed system.

Lemma 3.1 [15], [24]: Under the assumptions (A1)-(A3) and for $\mu$ sufficiently small, (3.3.4) has a unique hyperbolic periodic orbit $\left(p_{\mu}\left(t, t_{0}\right), \vartheta\left(t-t_{0}\right)\right)$. such that $p_{\mu}\left(t, t_{0}\right)=$ $p_{0}\left(t-t_{0}\right)+O(\mu)$. Correspondingly, the induced Poincaré map $P_{\mu}^{t_{0}}$ has a unique hyperbolic saddle point $p_{\mu}=p_{0}+$ $O(\mu)$.

Lemma 3.2 [15], [26]: The local stable and unstable manifolds $W_{\mathrm{loc}}^{s}\left(p_{\mu}\left(t, t_{0}\right)\right)$, and, respectively, $W_{\mathrm{loc}}^{u}\left(p_{\mu}\left(t, t_{0}\right)\right)$ of the perturbed periodic orbit $p_{\mu}\left(t, t_{0}\right)$ are $C^{k}$ close, $k \geqslant 1$, to those of the unperturbed periodic orbit $\left(p_{0}(t)\right.$ Moreover, orbits $x_{\mu}^{s}\left(t ; t_{0}\right)$ and $x_{\mu}^{u}\left(t, t_{0}\right)$ lying respectively in $W^{s}\left(p_{\mu}\left(t, t_{0}\right)\right.$ and $W^{u}\left(p_{\mu}\left(t, t_{0}\right)\right)$ and based on the section $\Sigma^{t_{0}}$ can be expressed as follows; with uniform validity over 
their indicated respective time intervals

$$
\begin{array}{rl}
x_{\mu}^{u}\left(t, t_{0}\right)=\bar{x}_{0}\left(t-t_{0}\right)+\mu x^{1 u}\left(t, t_{0}\right)+ & O\left(\mu^{2}\right), \\
t & \in\left(-\infty, t_{0}\right] \\
x_{\mu}^{s}\left(t, t_{0}\right)=\bar{x}_{0}\left(t-t_{0}\right)+\mu x^{1 s}\left(t, t_{0}\right)+ & O\left(\mu^{2}\right), \\
t & t \in\left[t_{0}, \infty\right) .
\end{array}
$$

(For the proofs of Lemmas 3.1 and 3.2, see Greenspan and Holmes [15].)

Now we embark on the derivation of a measure of the separation of the stable, $W^{s}\left(p_{\mu}\right)$, and unstable, $W^{u}\left(p_{\mu}\right)$, manifolds of the perturbed system (3.3.4). The measure is the Melnikov integral.

In the 3-dimensional space $R^{2} \times R$, select a point $\bar{x}_{0}(0)$ on the intersection of $\Sigma^{t_{0}}$ with the homoclinic manifold $\bar{\Gamma}_{0} \times R$ (see Fig. 3(e)). Let $f^{L}\left(\bar{x}_{0}(0)\right)$ denote the normal to the tangent space at $\bar{x}_{0}(0)$ (it is the vector $\left(-f_{2}\left(\bar{x}_{0}(0)\right), f_{1}\left(\bar{x}_{0}(0)\right)\right)^{T}$ for two-dimensional vector fields). Now consider the unique point on the manifold $W^{s}\left(p_{\mu}\right)$ (respectively, $W^{u}\left(p_{\mu}\right)$ ) at which the line in the direction of the vector $f^{L}\left(\bar{x}_{0}(0)\right)$, which is based at $\bar{x}_{0}(0)$, intersects this manifold with the following additional property:

This point of intersection is the "closest" (in the sense of elapsed time) to the perturbed hyperbolic point $p_{\mu}$ lying on the section $\Sigma^{t_{0}}$ (see points 1 and 2 in Fig. 3(f)).

Denote these two unique points $x_{\mu}^{u}\left(t_{0}\right)\left(:=x_{\mu}^{u}\left(t_{0}, t_{0}\right)\right)$ and $x_{\mu}^{s}\left(t_{0}\right)\left(=x_{\mu}^{s}\left(t_{0}, t_{0}\right)\right)$ belonging respectively to $W_{\mu}{ }^{u}\left(p_{\mu}\right)$ and $W_{\mu}^{s}\left(p_{\mu}\right)$. Thus one can define the separation between the stable and unstable manifolds as $d\left(t_{0}\right)=\mid x_{\mu}^{u}\left(t_{0}\right)-$ $x_{\mu}^{s}\left(t_{0}\right) \mid$ where $|\cdot|$ is the Euclidean norm. An improved definition which accounts for the "sign" of the separation is

$$
d\left(t_{0}\right)=\frac{f\left(\bar{x}_{0}(0)\right) \wedge\left[x_{\mu}^{u}\left(t_{0}\right)-x_{\mu}^{s}\left(t_{0}\right)\right]}{\left|f\left(\bar{x}_{0}(0)\right)\right|}
$$

which is the projection of the separation on the direction normal to the vector velocity $f\left(x_{0}(0)\right)$, see Fig. $3(\mathrm{f})$. The wedge product is defined by

$$
\left[\begin{array}{l}
a_{1} \\
a_{2}
\end{array}\right] \wedge\left[\begin{array}{l}
b_{1} \\
b_{2}
\end{array}\right]=a_{1} b_{2}-a_{2} b_{1}
$$

Utilizing Lemma 3.2 one can express the separation as

$$
d\left(t_{0}\right)=\mu \frac{f\left(\bar{x}_{0}(0)\right) \wedge\left(x^{1 u}\left(t_{0}, t_{0}\right)-x^{1 s}\left(t_{0}, t_{0}\right)\right)}{\left|f\left(\bar{x}_{0}(0)\right)\right|}+O\left(\mu^{2}\right) .
$$

To obtain all terms of the expansion, one requires that the functions $f$ and $g$ of the differential equation (3.3.4) be analytic. Then

$$
d\left(t_{0}\right)=\sum_{j=1}^{\infty} \mu^{j} \frac{f\left(\bar{x}_{0}(0)\right) \wedge\left(x^{j u}\left(t_{0}, t_{0}\right)-x^{j s}\left(t_{0}, t_{0}\right)\right)}{\left|f\left(\bar{x}_{0}(0)\right)\right|}
$$

which converges over some neighborhood.

To arrive at the Melnikov integral we derive the cquations for the first variation from (3.3.4) employing (3.3.6), to obtain

$$
\begin{aligned}
& \frac{d}{d t}\left(\bar{x}_{0}+\mu x^{1 u}+O\left(\mu^{2}\right)\right) \\
& \quad=f\left(\bar{x}_{0}+\mu x^{1 u}+O\left(\mu^{2}\right)\right)+\mu g\left(\bar{x}_{0}+\mu x^{1 u}+O\left(\mu^{2}\right), t\right) \\
& \quad=f\left(\bar{x}_{0}\right)+D_{x_{0}} f\left(\bar{x}_{0}\right) \cdot \mu x^{1 u}+\mu g\left(\bar{x}_{0}, t\right)+O\left(\mu^{2}\right)
\end{aligned}
$$

or

$$
\dot{x}^{1 u}=D_{x_{0}} f\left(\bar{x}_{0}\right) x^{1 u}+g\left(\bar{x}_{0}, t\right), \quad t \leqslant t_{0}
$$

as the differential equation for the first variational $x^{1 u}$. Similarly for $x^{1 s}$ we obtain

$$
\dot{x}^{1 s}=D_{x_{0}} f\left(\bar{x}_{0}\right) x^{1 s}+g\left(\bar{x}_{0}, t\right), \quad t \geqslant t_{0} .
$$

Remark: One can similarly obtain ordinary differential equations for the higher variations and thus proceed to derive an improved measure. This is so since one considers all terms of the expansion of the separation function $d\left(t_{0}\right)$. One may also obtain any finite number of terms of the expansion. But the improved accuracy is gained at the expense of simplicity of analytic evaluation.

To evaluate the first variation of the separation, we must determine the constant vectors $x^{1 u}\left(t_{0}, t_{0}\right)$ and $x^{1 s}\left(t_{0}, t_{0}\right)$. One may utilize the differential equation (3.3.9) which is defined uniformly on the time interval $\left(-\infty, t_{0}\right]$. We know a priori that the solution trajectory, beginning at $x^{1 u}\left(t_{0}, t_{0}\right)$ and evolving backward in time, converges exponentially fast to the perturbed saddle point $p_{\mu}$ lying on the section $\Sigma^{t_{0}}$ (see [15], [24]). The same convergence property holds for trajectories on the stable manifold and evolving forward in time. It then easily follows that, if $p_{\mu}$ is known, the constant vectors $x^{1 u}$ and $x^{1 s}$ can be determined. Our problem, therefore, is a form of the final (or initial) value problem.

Utilizing equation (3.3.8), we make the following definitions. $\Delta^{u}\left(t_{0}, t_{0}\right)=f\left(\bar{x}_{0}(0)\right) \wedge x_{\mu}^{1 u}\left(t_{0}, t_{0}\right)$ and $\Delta^{s}\left(t_{0}, t_{0}\right):=$ $f\left(\bar{x}_{0}(0)\right) \wedge x_{\mu}^{1 s}\left(t_{0}, t_{0}\right)$. Then we define the Melnikov function

$$
\Delta\left(t_{0}, t_{0}\right):=\Delta^{u}\left(t_{0}, t_{0}\right)-\Delta^{s}\left(t_{0}, t_{0}\right)
$$

and (3.3.8) can now be written as

$$
d\left(t_{0}\right)=\frac{\mu}{\left|f\left(\bar{x}_{0}(0)\right)\right|} \Delta\left(t_{0}, t_{0}\right)+O\left(\mu^{2}\right) .
$$

We seek to write the Melnikov function in terms of the given vector field to facilitate computations. To do so, we derive a differential equation for $\Delta^{u}$ (similarly $\Delta^{s}$ ), which is valid over the time interval $\left(-\infty, t_{0}\right]$. Compute the following time derivative (dropping arguments for simplicity)

$$
\frac{d}{d t} \Delta^{u}\left(t, t_{0}\right)=\dot{f} \wedge x^{1 u}+f \wedge \dot{x}^{1 u}
$$

using (3.3.9) one obtains

$$
\begin{aligned}
\dot{\Delta}^{u} & =D f_{x_{0}} f \wedge x^{1 u}+f \wedge\left[D f_{x_{0}} x^{1 u}+g\left(\bar{x}_{0}, t\right)\right] \\
& =\left(\operatorname{trace} D f_{x_{0}}\left(\bar{x}_{0}\right)\right) \Delta^{u}+f\left(\bar{x}_{0}\right) \wedge g\left(\bar{x}_{0}, t\right) .
\end{aligned}
$$

This is a scalar differential equation in $\Delta^{u}\left(t, t_{0}\right)$, and we 
note that

$$
\Delta^{u}\left(-\infty, t_{0}\right)=f\left(\bar{x}_{0}(-\infty)\right) \wedge x^{u}\left(-\infty, t_{0}\right) \equiv 0
$$

since $\bar{x}_{0} \rightarrow p_{0}$ as $t \rightarrow-\infty$. Integrating (3.3.12) over the domain of definition from $t=-\infty$ to $t=t_{0}$, and observing the exponential convergence property, results in

$$
\begin{aligned}
\Delta^{u}\left(t_{0}, t_{0}\right)=\Delta^{u}\left(t_{0}\right)= & \int_{-\infty}^{t_{0}} f\left(\bar{x}_{0}\left(t-t_{0}\right)\right) \wedge g\left(\bar{x}_{0}\left(t-t_{0}\right) ; t\right) \\
& \cdot \exp \left[-\int_{0}^{t-t_{0}} \operatorname{tracc} D f\left(\bar{x}_{0}(s)\right) d s\right] d t .
\end{aligned}
$$

Similarly one obtains an analogous result for $\Delta^{s}\left(t_{0}, t_{0}\right)$. Thus (3.3.11) becomes

$$
\begin{aligned}
\Delta\left(t_{0}\right)=\int_{-\infty}^{\infty} f\left(\bar{x}_{0}\left(t-t_{0}\right)\right) & \wedge g\left(\bar{x}_{0}\left(t-t_{0}\right) ; t\right) \\
\cdot \exp & {\left[-\int_{0}^{t-t_{0}} \operatorname{trace} D f\left(\bar{x}_{0}(s)\right) d s\right] d t . }
\end{aligned}
$$

This integral was rederived by Holmes (see also [24], [15], [26]) and is named the Melnikov integral. We remark that if the unperturbed vector field $f$ is that of a Hamiltonian $H_{0}$ then trace $D f\left(\bar{x}_{0}(s)\right)$ vanishes. Further assuming that the perturbation vector $g$ is also of another Hamiltonian, $H_{1}$, then the Melnikov integral reduces to

$$
\Delta\left(t_{0}\right)=\int_{-\infty}^{\infty}(f \wedge g) d t=-\int_{-\infty}^{\infty}\left\{H_{0}, H_{1}\right\} d t
$$

where the quantity $\left\{H_{0}, H_{1}\right\}$ is the Poisson bracket. Finally the following theorem (see [24], 15], [26]) gives conditions under which the Melnikov integral can serve as a measure of the presence of chaos.

Theorem 3.3.2: The unstable manifold $W^{u}\left(p_{\mu}\left(t_{0}, t_{0}\right)\right)$ and the stable manifold $W^{s}\left(p_{\mu}\left(t_{0}, t_{0}\right)\right)$ intersect transversely for sufficiently small $\mu>0$, if there exists at least one transversal zero, i.e., there is a $t_{0}^{\prime}$ such that $\Delta\left(t_{0}^{\prime}\right)=0$, and $\left(d / d t_{0}\right) \Delta\left(t_{0}^{\prime}\right) \neq 0$. Moreover if $\Delta\left(t_{0}\right)$ is bounded away from zero, then $\boldsymbol{W}^{u}\left(p_{\mu}\left(t_{0}, t_{0}\right)\right) \cap \boldsymbol{W}^{s}\left(p_{\mu}\left(t_{0}, t_{0}\right)\right)=\varnothing$.

An extension to higher dimensions can be made by considering a system composed of a cross product of $n$ subsystems each possessing a homoclinic orbit associated with a hyperbolic saddle. The combined system will have a $2 n$-dimensional phase space embedding an $n$-dimensional homoclinic manifold connecting a saddle point to itself. A perturbation can thus be applied to produce transversal intersection of stable and unstable manifolds of the perturbed saddle. This extension was employed by Gruendler utilizing the spherical pendulum as an example [16]. One can conclude that horseshoes are present within projections on the phase plane associated with each subsystem.

Another extension is to consider the coupling of a cross product of a subsystem possessing a homoclinic orbit with several subsystems, each of which is simply a nonlinear oscillator. This leads to a new phenomenon, namely, Arnold diffusion.

\section{ARnOld Diffusion AND THE Melnikov METHOD}

Arnold [5] introduced a new concept of instability in a specific example of a Hamiltonian system, a weakly cou- pled (i.e., nearly integrable) time-periodic two-degreeof-freedom Hamiltonian system. One degree of freedom possesses a homoclinic orbit, the second is a nonlinear oscillator and the weak coupling term is time periodic. We shall present a physical interpretation of Arnold diffusion, then introduce an adaptation of Arnold's result developed by Holmes and Marsden [27] to $(n>3)$ degree of freedom Hamiltonian systems. In Section III we extend the results to certain non-Hamiltonian systems.

\subsection{Meaning and Physical Insights}

Arnold diffusion is a self-generated "stochastic" motion that can occur in nearly integrable $n$-degree of freedom Hamiltonian systems where $n>3$. The integrable system underlying the system often possesses action-angle coordinates. In action space, the region of initial conditions generating stochastic motion is everywhere dense. For a fixed energy, this dense region amounts to what had been named the Arnold web. The stochastic and nonstochastic (regular) trajectories are intimately comingled, with some stochastic ones intersecting every region on the constant energy surface. Projecting the trajectories on the phase space of any degree of freedom, one observes that some projected trajectories travel to higher (or lower) energy levels of this degree of freedom. They do so crossing regular KAM (Kolmogrov-Arnold-Moser) curves (tori projected to the phase space of the degree of freedom). This phenomenon is an intrinsic property of Arnold diffusion and is not present in planar chaotic motion. In the two-degree-of-freedom case (four-dimensional state space) the regular KAM surfaces, residing in the constant energy hypersurface of dimension $3(=4-1)$, are two dimensional. Thus the KAM surfaces are of codimension 1 of the constant energy hypersurface. This means that the KAM surfaces partition the constant energy space into two separate pieces, forcing trajectories to reside only in one piece or the other. In general the constant energy hypersurface is of dimension $2 n-1$, and the regular KAM surfaces are of dimension $n$, where $n$ is the number of degrees of freedom. If $n \geqslant 3$, the KAM surfaces are not of codimension one relative to a constant energy hypersurface and thus cannot partition it. This permits, in principle, trajectories to exist which intersect any region of the constant energy space.

Chirikov [12] and others (see [12], [31] and references therein) have conducted simulations which confirm the presence of Arnold diffusion in many nearly integral Hamiltonian systems. They calculated the (Arnold) diffusion coefficients for several examples (see Lieberman [31], Lichtenberg and Lieberman [32], and [12]). A theoretical upper bound on the diffusion rate, for different degrees of freedom, has been calculated by Nekhoroshev (see [12], [32] and [6, p. 407]).

The experimental work is considerably ahead of theory. Other types of diffusion have been verified experimentally such as the one due to the overlapping of resonances (see [6], [32]). In the physics literature this diffusion is called strong stochasticity. It admits relatively large perturbation and occurs in two or more degrees of freedom. The over- 
lapping of resonances has not yet been explicitly dealt with via the Melnikov approach.

\subsection{Arnold Diffusion in Hamiltonian Systems}

In this section we summarize the results of Holmes and Marsden [27] for Hamiltonian systems with $n$-degree of freedom $(n \geqslant 3)$.

\section{Problem Statement}

Consider the unperturbed Hamiltonian system

$$
H^{0}(q, p, \vec{x}, \vec{y})=F(q, p)+G(\vec{x}, \vec{y}) .
$$

Here $F$ is a Hamiltonian which possesses a homoclinic orbit $(\bar{q}, \bar{p})$ associated with a hyperbolic saddle point $q_{0}, p_{0}$. Let $\bar{h}$ be the energy constant of this orbit, i.e., $F(\bar{q}, \bar{p})=\bar{h}$. The parameters $(q, p, \vec{x}, \vec{y})$ are canonical coordinates on a $2(n+1)$-dimensional symplectic manifold $\boldsymbol{P}$, where $q$ and $p$ are real and $\vec{x}=\left(x_{1}, \cdots, x_{n}\right), \vec{y}=\left(y_{1}, \cdots, y_{n}\right)$ are $n$ vectors. We assume that in a certain region of the state space a canonical transformation to action-angle coordinates $\left(\vartheta_{1}, \cdots, \vartheta_{n}, I_{1}, \cdots, I_{n}\right)$ can be found such that the system (4.2.1) takes the form

$$
H^{0}(q, p, \vec{\vartheta}, \vec{I})=F(q, p)+\sum_{i=1}^{n} G_{i}\left(I_{i}\right)
$$

where $G_{j}(0)=0$ for all $j$ and

$$
\Omega_{j}\left(I_{j}\right)=\frac{\partial G_{j}}{\partial I_{j}}>0 \quad \text { for } I_{j}>0 .
$$

Applying the reduction procedure (see Holmes and Marsden [26], [27]), we eliminate the action $I_{n}$ and replace the time variable by the $2 \pi$-periodic angle $\vartheta_{n}$. Then the equations

$$
\begin{aligned}
G_{j}\left(I_{j}\right) & =h_{j} \\
\vartheta_{j} & =\Omega_{j}\left(I_{j}\right) \vartheta_{n}+\vartheta_{j}(0), \quad j=1, \cdots, n-1 \\
q & =q_{0}, \quad p=p_{0}
\end{aligned}
$$

describe an (n-1)-parameter family of invariant $(n-1)$ dimensional tori $\boldsymbol{T}\left(h_{1}, \cdots, h_{n-1}\right)$. For a fixed set of $h_{1}, \cdots, h_{n-1}$, the torus $T\left(h_{1}, \cdots, h_{n-1}\right)$ is connected to itself by the $n$-dimensional homoclinic manifold

$$
\begin{aligned}
& G_{j}\left(I_{j}\right)=h_{j} \\
& \vartheta_{j}=\Omega_{j}\left(I_{j}\right) \vartheta_{n}+\vartheta_{j}^{0}, \quad 1 \leqslant j \leqslant n-1 \\
& q=\bar{q}\left(\vartheta_{n}-\vartheta_{n}^{0}\right), \quad p=\bar{p}\left(\vartheta_{n}-\vartheta_{n}^{0}\right)
\end{aligned}
$$

This manifold consists of the coincident stable and unstable manifolds of the torus $\boldsymbol{T}\left(h_{1}, \cdots, h_{n-1}\right)$, i.e.,

$$
\boldsymbol{W}^{s}\left(\boldsymbol{T}\left(h_{1}, \cdots, h_{n-1}\right)\right)=\boldsymbol{W}^{u}\left(\boldsymbol{T}\left(h_{1}, \cdots, h_{n-1}\right)\right) .
$$

The perturbed problem considered here has the following form:

$$
\begin{aligned}
H^{\mu}(q, p, \vec{\vartheta}, \vec{I})=F(q, p)+\sum_{i=1}^{n} & G_{i}\left(I_{i}\right) \\
& +\mu H^{\mathbf{l}}(q, p, \vec{\vartheta}, \vec{I})
\end{aligned}
$$

wherc $H^{1}$ is $2 \pi$-periodic in $\vartheta_{1}, \cdots, \vartheta_{n}$ and $\mu>0$. For suf- resonance and non-degeneracy conditions given below) a positive measure of the $(n-1)$-dimensional tori $\boldsymbol{T}\left(h_{1}, \cdots, h_{n-1}\right)$ persists (see Arnold [6, appendix 8]). We denote these tori by $\boldsymbol{T}_{\mu}\left(h_{1}, \cdots, h_{n-1}\right)$. Their corresponding stable and unstable manifolds $\boldsymbol{W}^{s}\left(\boldsymbol{T}_{\mu}\right)$, respectively, $\boldsymbol{W}^{u}\left(\boldsymbol{T}_{\mu}\right)$, are $C^{k}$ close, $k \geqslant 1$, to the unperturbed homoclinic manifold $\boldsymbol{W}^{s}\left(\boldsymbol{T}\left(h_{1}, \cdots, h_{n-1}\right)\right) \cap \boldsymbol{W}^{u}\left(\boldsymbol{T}\left(h_{1}, \cdots, h_{n-1}\right)\right)$.

Let $h>\bar{h}$ be the total energy of the perturbed Hamiltonian $H^{\mu}$ of equation (4.2.6). Now consider the $n$-parameter family of orbits filling the unperturbed homoclinic manifold. Let

$$
\begin{aligned}
\left(\bar{q}, \bar{p}, \vartheta_{1}, \cdots, \vartheta_{n}, I_{1}, \cdots, I_{n}\right) & =\left(\bar{q}(t), \bar{p}(t), \Omega_{1}\left(I_{1}\right) t\right. \\
& \left.+\vartheta_{1}^{0}, \cdots, \Omega_{n}\left(I_{n}\right) t+\vartheta_{n}^{0}, I_{1}, \cdots, I_{n}\right)
\end{aligned}
$$

be the parameterization of these orbits and select one. Let $\left\{F, H^{1}\right\}$ denote the $(q, p)$ Poisson bracket of $F(q, p)$ and $H^{1}\left(q, p, \vartheta_{1}, \cdots, \vartheta_{n}, I_{1}, \cdots, I_{n}\right)$ evaluated on this orbit. Similarly let

$$
\left\{I_{k}, H^{1}\right\}=-\frac{\partial H^{1}}{\partial \vartheta_{k}}, \quad k=1, \cdots, n-1
$$

be evaluated on the same orbit. Then define the Melnikov vector $\boldsymbol{M}\left(\vec{\vartheta}^{0}\right)=\left(\boldsymbol{M}_{1}, \cdots, \boldsymbol{M}_{n-1}, \boldsymbol{M}_{n}\right)$ by

$$
\begin{array}{r}
\boldsymbol{M}_{k}\left(\vartheta_{1}^{0}, \cdots, \vartheta_{n}^{0}, h, h_{1}, \cdots, h_{n-1}\right):=\int_{-\infty}^{\infty}\left\{I_{k}, H^{1}\right\} d t, \\
k=1, \cdots, n-1
\end{array}
$$

and

$$
\boldsymbol{M}_{n}\left(\vartheta_{1}^{0}, \cdots, \vartheta_{n}^{0}, h, h_{1}, \cdots, h_{n-1}\right):=\int_{-\infty}^{\infty}\left\{F, H^{1}\right\} d t
$$

where the integrals above are required to be convergent for suitable limits; i.e., $\int_{-\infty}^{\infty}$ means $\lim _{L \rightarrow \infty} \int_{-S_{n}}^{T_{n}}$ for suitable sequences $T_{n} \rightarrow \infty, S_{n} \rightarrow \infty$. If the limit exists, then the integrals are conditionally convergent.

Consider the following conditions:

(C1) $F$ possesses a homoclinic orbit $(\bar{q}(t), \bar{p}(t))$ connecting a saddle point $\left(q_{0}, p_{0}\right)$ to itself. Let $\bar{h}$ be the energy of this orbit.

(C2) $\Omega_{j}\left(I_{j}\right)=G_{j}^{\prime}\left(I_{j}\right)>0$ for $j=1, \cdots, n$.

(C3) The constants $G_{j}\left(I_{j}\right)=h_{j}, j=1, \cdots, n$ are chosen such that the unperturbed frequencies $\Omega_{1}\left(I_{1}\right), \cdots, \Omega_{n}\left(I_{n}\right)$ satisfy the non-degeneracy conditions (i.e., $\Omega_{j}^{\prime}\left(I_{j}\right) \neq 0, j=$ $1, \cdots, n)$ and the nonresonance condition, i.e., the equation

$$
\sum_{i=1}^{n} k_{i} \Omega_{i}\left(I_{i}\right)=0
$$

where $k_{i}$ are integers, implies $k_{i}=0$ for all $1 \leqslant i \leqslant n$.

(C4) The multiple $2 \pi$-periodic Melnikov vector $M: R^{n}$ $\rightarrow R^{n}$ has at least one transversal zero, i.e., a point $\left(\vartheta_{1}^{, 0}, \cdots, \vartheta_{n}^{, 0}\right)$ such that

$$
\boldsymbol{M}\left(\boldsymbol{\vartheta}_{1}^{, 0}, \cdots, \vartheta_{n}^{\prime 0}\right)=0 \text { and } \operatorname{det}\left[\boldsymbol{D M}\left(\boldsymbol{\vartheta}_{1}^{\prime 0}, \cdots, \vartheta_{n}^{\prime 0}\right)\right] \neq 0 \text {, }
$$

where $\boldsymbol{D M}$ is the $n \times n$ Jacobian matrix of the vector $\boldsymbol{M}$ with respect to the initial phases $\left(\vartheta_{1}^{0}, \cdots, \vartheta_{n}^{0}\right)$.

Finally we state the main result.

Theorem 4.1 [Holmes \& Marsden]: If conditions (C1)(C4) hold for the perturbed system (4.2.6) then, for $\mu$ 
folds $W^{s}\left(T_{\mu}\right)$ and $W^{u}\left(T_{\mu}\right)$ of the perturbed torus $T_{\mu}$ intersect transversally. Moreover, a finite transition chain of such tori $\boldsymbol{T}_{\mu}^{1}, \cdots, \boldsymbol{T}_{\mu}^{k}$ can be chosen; i.e., tori such that $\boldsymbol{W}^{u}\left(\boldsymbol{T}_{\mu}^{j}\right)$ 不 $\boldsymbol{W}^{s}\left(\boldsymbol{T}_{\mu}^{j+1}\right)$ and $\boldsymbol{W}^{u}\left(\boldsymbol{T}_{\mu}^{j+1}\right)$ 历 $\boldsymbol{W}^{s}\left(\boldsymbol{T}_{\mu}^{j}\right), 1 \leqslant j \leqslant k$ -1 .

The transition chain of tori are responsible for the occurrence of Arnold diffusion. Holmes and Marsden suggest that these transition tori can survive the addition of certain positive and negative damping, employing a technique which they had developed in [26].

An example which illustrates Theorem 4.1 is that of a simple pendulum linearly coupled to two nonlinear oscillators. Its perturbed Hamiltonian function can be written as follows (with the two oscillators in action-angle variables):

$$
\begin{aligned}
H^{\mu}= & \frac{p^{2}}{2}-\cos q+G_{1}\left(I_{1}\right)+G_{2}\left(I_{2}\right) \\
& +\left(\frac{\mu}{2}\right)\left[\left(\left(2 I_{1}\right)^{1 / 2} \sin \vartheta_{1}-q\right)^{2}+\left(\left(2 I_{2}\right)^{1 / 2} \sin \vartheta_{2}-q\right)^{2}\right] .
\end{aligned}
$$

Remark: The transition chain is reminiscent of the $\Omega$ decomposition theorem of Section II. The latter yields a more general type of diffusion. This connection has not been revealed nor explored yet. One would hope to establish that the concept of transition chain of tori constitutes a special case of the $\Omega$-decomposition theorem.

\subsection{Extension to $2 n$-Dimensional Non-Hamiltonian Systems}

Many physical dynamical systems are described by a set of ordinary differential equations. In this section we extend Theorem 4.1 to a certain class of non-Hamiltonian systems. tem:

We begin by considering the following perturbed sys-

$$
\dot{x}=f(x)+\mu g(x, t), \quad x \in R^{2 n}
$$

where

$$
x=\left(x_{0}, x_{1}, x_{2}, \cdots, x_{n-1}\right), \quad x_{i} \in R^{2}
$$

and $f$ and $g$ have the form

$$
\begin{array}{cc}
f(x)=\left[\begin{array}{c}
f_{0}\left(x_{0}\right) \\
\vdots \\
f_{n-1}\left(x_{n-1}\right)
\end{array}\right], & \text { where } f_{i}: R^{2} \rightarrow R^{2}, \\
g(x, t)=\left[\begin{array}{c}
g_{0}(x, t) \\
\vdots \\
g_{n-1}(x, t)
\end{array}\right], & \text { for all } i=0, \cdots, n-1
\end{array}
$$

Assume $f$ and $g$ to be sufficiently smooth $\left(C^{k}, k \geqslant 2\right)$ and bounded on bounded sets. Also assume $g_{i}(x, t)$ is $T$-periodic in $t$. Setting $\mu=0$, we obtain the unperturbed system on $R^{2 n}$ as

$$
\dot{x}_{i}=f_{i}\left(x_{i}\right), \quad i=0, \cdots, n-1
$$

Assume that (4.3.2) possess a homoclinic manifold con- a homoclinic orbit which is a cross product of solutions of the following subsystems $(0 \leqslant j \leqslant n-1)$ :

$$
\dot{x}_{j}=f_{j}\left(x_{j}\right)
$$

We state the following assumptions which resemble those in the previous section.

(A1) The unperturbed subsystem (4.3.2.0) (i.e., $\dot{x}_{0}=$ $\left.f_{0}\left(x_{0}\right)\right)$ : (A1.a) possesses a homoclinic orbit $\bar{x}_{0}(t)$ associated with a hyperbolic saddle point $p_{0}$ (one may similarly consider a heteroclinic orbit). Let

$$
\bar{\Gamma}^{0}=\left\{\bar{x}_{0}(t) \mid t \in R\right\} \cup\left\{p_{0}\right\} .
$$

(A1.b) Solutions of (4.3.2.0) exist and are defined within some compact region $\bar{D}$ in $R^{2}$ containing $\bar{\Gamma}^{0}$.

(A2) Each unperturbed subsystem (4.3.2.j), $1 \leqslant j \leqslant n-1$, is Hamiltonian and there exists a transformation to action-angle coordinates, i.e.,

$$
\begin{aligned}
& \Theta_{j}=\Omega_{j}\left(I_{j}\right), \quad \Omega_{j}\left(I_{j}\right)>0 \text { for } I_{j}>0 \\
& \dot{I}_{j}=0
\end{aligned}
$$

where $\Omega_{j}\left(I_{j}\right)$ are parametrized by the action $I_{j}$ and satisfy the nondegeneracy and nonresonance conditions cited in the last section.

(A3) $g(x, t)=\left(g_{0}(x, t), \cdots, g_{n-1}(x, t)\right)^{t}$ is a Hamiltonian vector field with an energy function $H^{1}(x, t)$.

Define the following Melnikov integrals along trajectories of the unperturbed homoclinic manifold.

$$
\begin{aligned}
\boldsymbol{M}_{n}\left(t_{1}, t_{2}, \cdots, t_{n-1}, t_{n}\right) \\
:=\int_{-\infty}^{\infty} f_{0}\left(\bar{x}_{0}(t)\right) \wedge g_{0}\left(\bar{x}\left(t-t^{*}\right), t-t_{n}\right) \\
\cdot \exp \left\{-\int_{0}^{t} \operatorname{tr}\left\{\frac{\partial}{\partial x} f_{0}\left(\bar{x}_{0}(s)\right)\right\} d s\right\} d t
\end{aligned}
$$

where

$$
\bar{x}\left(t-t^{*}\right):=\left(\bar{x}_{0}(t), \bar{x}_{1}\left(t-t_{1}\right), \cdots, \bar{x}_{n-1}\left(t-t_{n-1}\right)\right) .
$$

Similarly, define the following integrals for $1 \leqslant k \leqslant n-1$.

$$
\begin{aligned}
\boldsymbol{M}_{k}\left(t_{1}, t_{2}, \cdots, t_{n-1}, t_{n}\right) & =\int_{-\infty}^{\infty} f_{k}\left(\bar{x}_{k}\left(t-t_{k}\right)\right) \\
\wedge & g_{k}\left(\bar{x}\left(t-t^{*}\right), t-t_{n}\right) d t .
\end{aligned}
$$

(A4) Let

$$
\boldsymbol{M}\left(t_{1}, \cdots, t_{n}\right):=\left(\boldsymbol{M}_{1}\left(t_{1}, \cdots, t_{n}\right), \cdots, \boldsymbol{M}_{n}\left(t_{1}, \cdots, t_{n}\right)\right)
$$

and assume that it has, at least, one transversal zero, i.e., there is a point $\left(t_{1}^{\prime}, \cdots, t_{n}^{\prime}\right)$ such that

$$
\boldsymbol{M}\left(t_{1}^{\prime}, \cdots, t_{n}^{\prime}\right)=0 \text { and } \operatorname{det}\left[\boldsymbol{D} \boldsymbol{M}\left(t_{1}^{\prime}, \cdots, t_{n}^{\prime}\right)\right] \neq 0
$$

where $\boldsymbol{D M}$ is, as before, the $n \times n$ Jacobian matrix.

Define $\boldsymbol{T}_{\mu}, \boldsymbol{W}^{s}\left(\boldsymbol{T}_{\mu}\right)$ and $\boldsymbol{W}^{u}\left(\boldsymbol{T}_{\mu}\right)$ as before, for a proof of the next result see [2].

Theorem 4.2: If conditions (A1)-(A4) hold for the system (4.3.1) then, for $\mu$ sufficiently small, the perturbed stable and unstable manifolds $W^{s}\left(T_{\mu}\right)$ and $W^{u}\left(T_{\mu}\right)$ of the perturbed torus $\boldsymbol{T}_{\mu}$ intersect transversally. There exist also transition chains.

\section{ACKNOWLEDGMENT}

The authors thank P. Holmes for inspiration and F. Wu 


\section{REFERENCES}

[1] F. M. Abdel Salam, "Analytic expressions of the unstable manifold of dynamical systems of differential equations," Master thesis in mathematics, University of California, Berkeley, Dec. 1982.

[2] "Chaos and Arnold diffusion in dynamical systems with applications to power systems," Ph.D. thesis, University of California, Berkeley, Dec. 1982.

[3] R. Abraham, and I. E. Marsden, Foundations of Mechanics. Reading, MA: 2nd Edition, Benjamin, 1978.

[4] A. A. Andronov, and C. E. Chaikin, Theory of Oscillations. Princeton, N.J.: Princeton Univ. Press, 1949

[5] V. I. Arnold, "Instability of dynamical systems with several degrees of freedom," Dokl. Akad. Nauk. SSSR 156, pp. 9-12, 1964.

[6] Mathematical Methods of Classical Mechanics. BerlinHeidelberg: Springer-Verlag, 1978.

[7] N. P. Bhatia and G. P. Szego, Stability Theory of Dynamical Systems. Berlin-Heidelberg: Springer-Verlag, 1970.

[8] G. D. Birkhoff, Dynamical Systems A.M.S. Publications, Providence, R.I. 1927.

[9] W. M. Boothby, An Introduction to Differentiable Manifolds and Riemannian Geometry Académic Press, 1975

[10] Brockett, R. W., "On the asymptotic properties of solutions of differential equations with multiple equilibria," J. Differential Equations, vol. 44, no. 2, pp. 249-262, May 1982.

[11] D. R. J. Chillingworth, Differential Topology with a View to Applications. London: Pitman, 1976.

[12] B. V. Chirikov, "A universal instability of many dimensional oscillator systems," Phys. Rep. 52, pp. 265-379, 1979

[13] S. N. Chow, J. K. Hale, and J. Mallet-Paret, "An example of bifurcation to homoclinic orbits," J. Differential Equations, vol. 37, pp. $351-373,1980$.

[14] J. Dieudonne, Foundations of Modern Analysis, Academic, 1960

[15] B. Greenspan and P. J. Holmes, "Homoclinic orbits, subharmonics and global bifurcations in forced oscillators," in Nonlinear Dynamics and Turbulence, Ed. G. Barenblatt, G. Iooss and D. D. Joseph, Pitman.

[16] J. Gruendler, Thesis, Math. Dep., Univ. North Carolina, 1981.

[17] J. Guckenheimer, "A strange, strange attractor" in, J. E. Marsden and M. McCracken (Eds.), The Hopf Bifurcation and Its Applications., New York: Springer-Verlag, 1976

[18] W. Hahn, Stability of Motion. Berlin-Heidelberg: Springer-Verlag, 1967.

[19] P. Hartman, Ordinary Differential Equations. New York: Wiley, 1964.

[20] B. D. Hassard, N. D. Kazarinoff, and Y-H. Wan, Theory and Applications of Hopf Bifurcation. London Math. Society, Lecture Note Series no. 41 Cambrige University Press, 1981.

[21] M. Henon, "A two dimensional mapping with a strange attractor" Commun. Math. Physics, vol. 50, pp. 69-77, 1976.

[22] M. W. Hirsch, C. C. Pugh, and M. Shub, Invariant Manifolds. Springer Lecture Notes in Mathematics no. 583, New York, Springer Verlag, 1977.

[23] M. W. Hirsch, and S. Smale, Differential Equations, Dynamical Systems, and Linear Algebra. New York, Academic Press. 1974.

[24] P. Holmes, "Averaging and chaotic motions in forced oscillations", SIAM. J. Appl. Math., vol. 38, 68-80 and 40, 167-168. 1980.

[25] P. Holmes and J. Marsden, "A partial differential equation with infinitely many periodic orbits: Chaotic oscillations of a forced beam", Arch. Rational Mechanics Anal., vol. 76, no. 2, pp. 135-166, 1981.

[26] two degrees of freedom" Commun. Math. Physics, vol. 82, pp. $523-544,1982$.

[27] , "Melnikov's method and Arnold diffusion for perturbations of integrable Hamiltonian systems," J. Math. Physics, vol. 23 no. 4, pp. $669-679,1982$

[28] _Horseshoes and Arnold diffusion for Hamiltonian systems on Lie groups", Indiana University Mathematical J., Apr. 1983.

[29] N. Kopell and R. B. Washburn, Jr., "Chaotic motions in the two degree-of-freedom swing equations", IEEE Trans. Circuits Syst, Nov. 1982

[30] O. Landford, Lecture notes Math. Depart. UCB 1981

[31] M. A. Lieberman, "Arnold diffusion in Hamiltonian systems with three degrees of freedom, "Ann. N.Y. Acad. Sci., vol. 357, pp. 119-142, 1980.

[32] A. J. Lichtenberg, and M. A. Lieberman, Regular and Stochastic Motion, Applied Math. Sciences, vol. 38, Springer-Verlag.

[33] A. I. Mees and C. T. Sparrow "Chaos" IEE Proceedings Vol. 128, Pt. D, No. 5, September 1981, pp. 201-205.

[34] J. W. Milnor, Topology from the Differentiable Viewpoint. The University Press of Virginia, Charlottesville, 1976.

[35] J. K. Moser, Stable and Random Motions in Dynamical Systems, with special emphasis on celestial mechanics, Ann. Math. Studies no. 77, Princeton Univ. Press, Princeton, N.Y. 1973.

[36] S. E. Newhouse, Lectures on Dynamical Systems in Dynamical Systems, C.I.M.E. Lectures Bressanone, Italy, June 1978, Progress in Mathematics \#8, Birkhouser, Boston Inc. 1980 .
[37] Nitecki, Z., Differentiable Dynamics. Cambridge MA: M.I.T. Press, 1971.

[38] Picard, Emile, Traite D'ANALYSE, Tome III. Gauthier-Villars Et Fils, Imprimeurs-Libraires, 1896.

[39] Pliss, V. A., Nonlocal Problems of the Theory of Oscillations. New York: Academic Press, 1966

[40] Smale, S., “Morse Inequalities for a Dynamical System", B.A.M.S. $66,43-49,1960$.

[41] Smale, S., "On Gradient Dynamical Systems", Ann. of Math. (2) vol. 74 (1961), pp. 199-206.

[42] Smale, S., "Differentiable Dynamical Systems", B.A.M.S. 73, 747-817, 1967.

[43] Sparrow, C. T., The Lorenz Attractor. Appli. Math. Sciences \# 42, Springer 1982.

[44] Sternberg, S. "Local contractions and a theorem of Poincare" Amer. J. Math. vol. 79, pp. 809-823, 1957.

[45] F. M. Abdel Salam, J. E. Marsden, and P. P. Varaiya, "Arnold diffusion in the swing equation of a power system," memo. UCBERL M83/13, Electronics Res. Lab., College of Eng., Univ. of California, Berkeley, CA 94720.

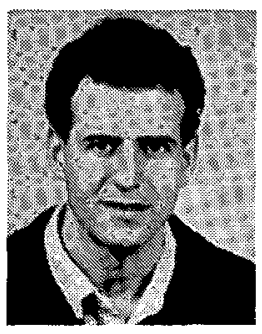

Fathi M. A. Salam received the M.S. degree in clectrical engincering from the University of California, Davis, in 1979, the B.S. and Ph.D. degrees in 1976 and 1982 , respectively, both in electrical engineering, and the M.A. degree in mathematics in 1982, from the University of California, Berkeley. From 1976 to Dec. 1977 he worked for a subsidiary of Exxon in the instrumentation and maintenance division.

$\mathrm{He}$ is currently a Visiting Assistant Professor of electrical engineering at the University of California at Berkeley. His areas of research interest are linear and nonlinear sustems, computational algorithms, complicated dynamics, applications of chaos and Arnold diffusion, stability and control of power systems, and interconnected large scale systems analysis.

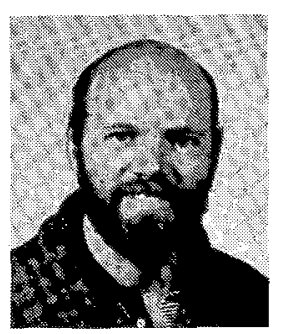

Jerrold E. Marsden received the B.Sc. degree from the University of Toronto and the Ph.D. degree in Mathematical Physics from Princeton University, NJ.

$\mathrm{He}$ is currently Professor of Mathematics at the University of California, Berkeley, has also taught in Canada, France, and Britain. Dr. Marsden received the Gravitation Research Prize in 1973, a Carnegie Fellowship in 1977, a Killam Fellowship in 1979 and held a Miller Professorship in 1981-82. Dr. Marsden's publications include the following books Applications of Global Analysis in Mathematical Physics (1974), The Hopf Bifurcation and its Applications (with M. McCracken, 1976), Foundations of Mechanics (with R. Abraham, 1978), A Mathematical Introduction to Fluid Mechanics (with A. Chorin, 1979), The Mathematical Foundations of Elasticity (with T. Hughcs, 1983), and Manifolds, Tensor Analysis and Applications (with R. Abraham and T. Ratiu, 1983).

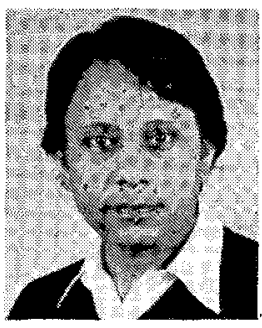

Pravin Varaiya received the B.S. degree from the University of Bombay, Bombay, India, and the M.S. and $\mathrm{Ph} . \mathrm{D}$. degrees from the University of California, Berkeley

He is now a Professor of Electrical Engineering and Computer Sciences and Economics at the University of California, Berkeley. He has been a Visiting Professor at M.I.T., Cambridge, and at the Federal University of Rio de Janiero, Brazil He was a Guggenheim Fellow in 1971 and a Miller Professor in 1979. His current interests are in power systems, adaptive control, and urban economics. 